\title{
Die Immunität der Mitglieder der Bundesversammlung
}

\author{
Helmut Winkelmann
}

Die Bundesversammlung ist in Art. 54 Abs. 3 GG vorgesehen, hat als einzige Aufgabe die Wahl eines Bundespräsidenten und setzt sich je zur Hälfte aus den Mitgliedern des Bundestages und einer gleichen Zahl von den Landesparlamenten gewählter Mitglieder zusammen, ohne dass diese dem jeweiligen Landesparlament angehören müssen.

\section{Gesetzesänderung und erster Anwendungsfall}

Am 20. Juli 2007 ist eine Änderung des Gesetzes über die Wahl des Bundespräsidenten durch die Bundesversammlung in Kraft getreten ${ }^{1}$, die den in $\$ 7$ Abs. 1 dieses Gesetzes auch für Mitglieder der Bundesversammlung verankerten Immunitätsschutz konkretisiert hat. Dieser war zwar schon 1959 einfachgesetzlich geschaffen worden ${ }^{2}$. Die Regelung hat aber, soweit ersichtlich, erstmals im Vorfeld der 12. Bundesversammlung am 23. Mai 2004 praktische Bedeutung erlangt.

$\$ 7$ in der 2004 geltenden Fassung beschränkte sich darauf, neben dem Zeugnisverweigerungsrecht und dem Behinderungsverbot (Art. 47, 48 Abs. 2 GG) auch Art. 46 GG und damit die Indemnität und Immunität der Bundestagsabgeordneten - auf die Mitglieder der Bundesversammlung entsprechend anwendbar sein zu lassen. Es existierten aber keine näheren Bestimmungen gesetzlicher, geschäftsordnungsrechtlicher oder sonstiger ${ }^{3}$ Natur zu Einzelfragen. So war ungeregelt, wer für Immunitätsangelegenheiten zuständig war und wann der Schutz für die von den Landesparlamenten Gewählten einsetzte.

Diese Ausgangslage fanden der Bundestag und sein Ausschuss für Wahlprüfung, Immunität und Geschäftsordnung (so genannter 1. Ausschuss) in der 15. Wahlperiode vor, als eine Staatsanwaltschaft beim Bundestag um Genehmigung nachsuchte, um gegen ein vom Berliner Abgeordnetenhaus gewähltes Mitglied der Bundesversammlung strafrechtliche Ermittlungen einleiten zu können. Auch das Schrifttum ergab für die Ausschussberatungen keine weiterführenden Hinweise, sondern erwähnte, wenn überhaupt, zumeist nur die gesetzliche Regelung. Soweit ausnahmsweise eine Begründung für die entsprechende Anwendung von Art. 46 GG erfolgte, wurde auf die Sicherstellung der Funktionsfähigkeit und des Ansehens der Bundesversammlung verwiesen ${ }^{4}$. Betont wurde auch, dass es sich bei Art. 46 GG um typische Rechte eines Parlamentariers handele, die ihrem Sinn und Zweck nach

1 Gesetz vom 12. Juli 2007, BGBl. I, S. 1326.

2 BGBl. I, S. 230, geändert durch Gesetz vom 24. Juni 1975 (BGBl. I, S. 1593).

3 Nummern $191 \mathrm{ff.}$ der Richtlinien für das Straf- und Bußgeldverfahren (RiStBV) mit Ausführungsbestimmungen zur Immunität der Mitglieder des Bundestages, der Landesparlamente und des Europäischen Parlaments erwähnen die Bundesversammlung nicht.

4 So bereits vor der gesetzlichen Regelung Eberhard Stöve, Die Bundesversammlung mit dem Entwurf eines Ausführungsgesetzes gemäß Artikel 54, Absatz 7 Grundgesetz, jur. Diss., Köln 1956, S. 33 . 
auch auf Mitglieder der Bundesversammlung anwendbar sein müssten ${ }^{5}$. Im Übrigen wurde angesichts der Zusammensetzung der Bundesversammlung eine Bedeutung entweder nur für diejenigen Personen angenommen, die nicht schon durch ein Mandat als Bundestagsoder Landtagsabgeordneter geschützt $\operatorname{sind}^{6}$, oder nur für solche Landesparlamentarier, deren landesrechtliche Immunität hinter der des Grundgesetzes zurückbleibt ${ }^{7}$. Auch die praktische Bedeutung wurde angesichts der Kürze der Zugehörigkeit zur Bundesversammlung bezweifelt ${ }^{8}$.

In seiner Beratung hat der Immunitätsausschuss für die von den Landesparlamenten Gewählten den Beginn des Immunitätsschutzes mit dem Erwerb der Mitgliedschaft in der Bundesversammlung angesetzt und nicht erst mit dem Zusammentritt der Bundesversammlung. Sodann wurde eine Zuständigkeit des Bundestages bejaht und damit eine solche der Bundesversammlung beziehungsweise eines ihrer Organe oder des betreffenden Landesparlaments verneint. Für dieses Beratungsergebnis waren mehrere Gesichtspunkte maßgebend: Handlungsfähigkeit der Bundesversammlung erst bei ihrem Zusammentritt; Verweisung in $\$ 7$ auf Art. 46 GG mit dort auch festgehaltener Bundestagszuständigkeit sowie Parallele zur ungeschriebenen Bundestagszuständigkeit für Immunitätsangelegenheiten des Bundespräsidenten gemäß Art. 60 Abs. 4 GG? .

Das Beratungsergebnis ist in einem Bericht dokumentiert, der wegen der erstmaligen Entscheidungslage abweichend von der ansonsten geübten Praxis zur Erläuterung der Rechtslage der Beschlussempfehlung an das Plenum beigefügt wurde ${ }^{10}$. Dort wurde auch betont, dass es ebenso wie bei Verfahren gegen Bundestagsabgeordnete nicht möglich sei, Angaben zum Tatvorwurf und zur Ausschussberatung zu machen. Dies entspricht der langjährigen Praxis in Immunitätsangelegenheiten, im Umgang mit personenbezogenen Daten Zurückhaltung zu wahren, wie sie auch bei der anonymisierenden Gestaltung von Tagesordnungen, dem Verzicht auf eine Plenardebatte in Immunitätsangelegenheiten sowie der restriktiven Verteilung von Ausschussprotokollen zu beobachten ist.

Sodann hat der Bundestag aufgrund der Beschlussempfehlung im beantragten Fall ${ }^{11}$ sowie in der Folge in drei weiteren Fällen ${ }^{12}$ - die Einleitung von staatsanwaltlichen Ermittlungen genehmigt. Schon diese waren genehmigungsbedürftig, da die regelmäßig bei Übernahme der Geschäftsordnung in der konstituierenden Sitzung eines neugewählten Bundestages beschlossene generelle, nur eine Unterrichtung des Bundestages verlangende

5 Udo Fink, in: Hermann von Mangoldt / Friedrich Klein / Christian Starck (Hrsg.), Grundgesetz, Bd. 2, 4. Auflage, München 2000, Art. 54, Rn. 48.

6 Jürgen Jekewitz, in: Erhard Denninger / Wolfgang Hoffmann-Riem / Hans-Peter Schneider / Ekkehart Stein (Hrsg.), Alternativ-Kommentar zum Grundgesetz, Bd. 2, 3. Auflage, Neuwied 2001, Art. 54, Rn. 10; Beate Braun, Die Bundesversammlung, Frankfurt am Main 1993, S. 109.

7 Werner Beulke, in: Ewald Löwe / Werner Rosenberg, Strafprozessordnung, Bd. 3, 25. Auflage, Berlin $2001-2004, \$ 152 a$, Rn. 11.

8 Jürgen Jekewitz, a.a.O. (Fn. 6).

9 So zum Beispiel schon Roman Herzog, in: Theodor Maunz / Günter Dürig (Hrsg.), Kommentar zum Grundgesetz, München, Art. 60 (Bearbeitung Mai 1986), Rn. 59.

10 Beschlussempfehlung und Bericht des Ausschusses für Wahlprüfung, Immunität und Geschäftsordnung (1. Ausschuss) vom 2. Februar 2004 - Drucksache 15/2879.

11 Vgl. Stenografisches Protokoll der 103. Sitzung vom 2. Februar 2004, S. 9336 D.

12 Vgl. Drucksachen 15/3007, 3008 und 3107. 
Genehmigung zur Einleitung von Ermittlungen ${ }^{13}$ nur für Bundestagsabgeordnete gilt. Angesichts des Fehlens jeglicher Detailregelung wurden die Genehmigungen mit der Auflage versehen, dass die für Bundestagsabgeordnete geltenden Bestimmungen zu beachten sind. Hierbei handelt es sich neben dem in Fußnote 13 erwähnten Beschluss betreffend Aufhebung der Immunität, der außer der generellen Genehmigung weitere Verfahrensregelungen trifft, um die ebenfalls in Anlage 6 zur Geschäftsordnung wiedergegebenen Grundsätze des Ausschusses in Immunitätsangelegenheiten gemäß $\$ 107$ Abs. 2 GO-BT. Die angesprochenen Verfahren gaben zugleich Anlass, auf Ausschussebene über die gesetzliche Grundlage, insbesondere eine Konkretisierung, zu beraten, die schließlich in der 16. Wahlperiode zur jetzigen Gesetzesänderung geführt hat (vgl. hierzu näher unter 3.).

\section{Anlass des gesetzlichen Immunitätsschutzes}

Anhaltspunkte, warum ein Immunitätsschutz erstmals - und einfachgesetzlich - vor der 3. Bundesversammlung 1959 unter Ablösung rudimentärer Regelungen zur Bundesversammlung im Bundeswahlgesetz für den ersten und zweiten Bundestag festgelegt worden ist, haben sich nur außerhalb der Gesetzesmaterialien finden lassen. Für die 2. Bundesversammlung am 17. Juli 1954 hatte der Landtag Nordrhein-Westfalen die KPD-Mitglieder Walter Fisch $^{14}$ und Friedrich Rische ${ }^{15}$ gewählt, die kein Landtagsmandat innehatten. Beide befanden sich seinerzeit in Untersuchungshaft, konnten aber letztlich an der Bundesversammlung teilnehmen ${ }^{16}$. Bundestagspräsident Hermann Ehlers bat - möglicherweise von dritter Seite angestoßen ${ }^{17}$ - am 24. Juni 1954 telegraphisch den nordrhein-westfälischen Landtagspräsidenten um Stellungnahme zur Frage der Immunität „der vom Nordrhein-Westfälischen Landtag in die Bundesversammlung gewählten Wahlmänner Fisch und Rische, die zur Zeit inhaftiert sind"18. Geantwortet wurde, dass beide mangels Landtagszugehörigkeit keine Immunität genössen. Auf Nachfrage des Bundestagspräsidenten vom 25. Juni 1954, ob der Landtag Schritte unternehmen wolle, um den von ihm Gewählten die Teilnahme an der Bundesversammlung zu ermöglichen, da eine Entscheidung der Bundesversammlung zu spät käme, wurde seitens des Landtagspräsidenten auf die fehlende Legitimation des Landtages für einen Rechtsschutz der Wahlmänner verwiesen. Angeregt wurde aber, dem Untersuchungsrichter die Aussetzung des Haftbefehls für die Dauer der Wahlhandlung vorzuschlagen. Das ebenfalls befragte Bundesjustizministerium sah laut einer in Archivakten des Bundestages befindlichen „Notiz aus [dem] Bundesjustizministerium“ nach „einhelliger“ Auffassung („Minister ist auch befragt worden“) keinen Immunitätsschutz, machte

13 Nr. 1 des Beschlusses des Deutschen Bundestages betr. Aufhebung der Immunität von Mitgliedern des Bundestages (Anlage 6 zur Geschäftsordnung des Deutschen Bundestages [GO-BT]).

14 Geboren 1910, verstorben 1966; MdB in der ersten Wahlperiode.

15 Geboren 1914, verstorben 2007; MdB in der ersten Wahlperiode.

16 So ausdrücklich im Beitrag Die Wahl des Bundespräsidenten, in: Das Parlament vom 14. Juli 1954.

17 Laut Kölner Stadt-Anzeiger vom 10. Juli 1954 soll sich Heinz Renner (MdB der 1. WP; KPD) um einen „Urlaub auf Ehrenwort“ für die Inhaftierten bemüht und „in Gesprächen im Bundeshaus beteuert haben, die kommunistischen Wahlmänner würden von einem etwaigen Urlaub auf Ehrenwort ganz bestimmt in ihre Haftzellen zurückkehren“.

18 Quelle - auch für Folgendes -: Parlamentsarchiv des Deutschen Bundestages 5100 1/55,1. 
aber darauf aufmerksam, dass die beiden Gewählten beim Bundesgerichtshof unter Hinweis auf ihre „angebliche Immunität“ als Delegierte der Bundesversammlung eine Haftentlassung für diese Tage beantragt hätten. Der entsprechende Vermerk trägt den handschriftlichen Zusatz, dass sie durch Beschluss des Bundesgerichtshofs (BGH) vom 28. Juni 1954 aus der Haft entlassen worden seien. Dies bezieht sich auf die Entscheidung in einem Haftprüfungstermin. So beantragte der Verteidiger von Fisch und Rische vor dem 6. Strafsenat des BGH am 28. Juni 1954, die Vollstreckung der Haftbefehle unter einer Auflage und der Ausnahme auszusetzen, vom 15. bis 18. Juli die Bundesrepublik Deutschland zur Teilnahme an der Wahl des Bundespräsidenten in Berlin verlassen zu dürfen ${ }^{19}$. Der Oberbundesanwalt hatte zuvor einen verfassungsrechtlich oder gesetzlich geregelten Immunitätsschutz verneint. Der 6. Strafsenat hielt die Haftbefehle aufrecht, setzte den Vollzug aber unter anderem mit der Auflage aus, „das Gebiet der Bundesrepublik außer zur Teilnahme an der Bundesversammlung in Berlin nicht zu verlassen“. Zwar geht eine Dissertation von einer Intervention des Bundesverfassungsgerichts aus ${ }^{20}$; derartiges ist aber den durchgesehenen Unterlagen nicht zu entnehmen.

Die Vorgänge des Jahres 1954 werden in den Gesetzgebungsmaterialien der Regelung von 1959 allerdings nicht erwähnt. Die Begründung des Regierungsentwurfs erachtete es nur als notwendig, ,allen Mitgliedern der Bundesversammlung für deren Dauer Indemnität, Immunität, Zeugnisverweigerungsrecht und Mandatsschutz wie den Bundestagsabgeordneten zu gewähren, zumal zu Mitgliedern auch Personen gewählt werden könnten, die weder Abgeordnete des Bundestages noch eines Landtages sind“21. Weder in den Beratungen des Ausschusses für Inneres ${ }^{22}$, dem die Vorlage allein und ohne Aussprache vom Plenum überwiesen wurde, noch vor Einbringung im Rahmen einer Befassung des so genannten Wahlrechtsausschusses (1. Sonderausschuss der 2. Wahlperiode) wurde die Immunitätsgewährung thematisiert. Im Innenausschuss wurde nur für erforderlich gehalten, den Zeitpunkt des Endes der Bundesversammlung wegen der Bedeutung für die Rechtsstellung der Mitglieder festzulegen; dies mündete in die Bestimmung, dass der Bundestagspräsident nach Annahme der Wahl durch den erfolgreichen Kandidaten die Bundesversammlung ausdrücklich für beendet erklärt $(\$ 9 \text { Abs. } 5)^{23}$. Über Gründe, warum der konkrete Anlass

19 Aktenzeichen BJs 221/54, Bundesarchiv Koblenz, Aktenband B 362/4934.

20 Eberhard Stöve, a.a.O (Fn. 4), S. 33 - ohne Quellenangabe. Dass das Bundesverfassungsgericht (BVerfG) analog Art. 46 GG eine vorläufige Entlassung verfügt haben soll, dürfte wohl eine Verwechslung mit der fünf Monate späteren mündlichen Verhandlung im KPD-Verbotsverfahren sein. Dort hatte die KPD unter anderem Fisch und Rische mit ihrer Vertretung beauftragt. Rische soll in Untersuchungshaft, ein Haftbefehl gegen Fisch aus Gesundheitsgründen außer Vollzug gewesen sein. Der Berichterstatter des 1. Senats, Bundesverfassungsrichter Erwin Stein, bat den 6. Strafsenat unter anderem für Fisch um ,sicheres Geleit“. Bezüglich Rische sah er davon ab, zum Verlangen der KPD auf Aussetzung des Haftbefehls Stellung zu nehmen. Der BGH erteilte Fisch sicheres Geleit für die mündliche Verhandlung. Der Ermittlungsrichter des BGH gestattete Rische, sich im Gebäude des Bundesverfassungsgerichts ohne Überwachung mit Vertretern der $\mathrm{KPD}$ zu besprechen und an den Verhandlungen teilzunehmen (dokumentiert in BVerfGE 5, S. 85, S. 107 sowie ausführlich bei Gerd Pfeiffer / Hans-Georg Strickert, KPD-Prozess, Karlsruhe 1955/1956, Bd. 1, S. 91 f.).

21 Drucksache $3 / 358$ vom 30. April 1958, S. 5 (zu $₫ 6$ Abs. 1, textgleich mit dem späteren $₫ 7$ ).

22 Der seinerzeitige Ausschuss für Geschäftsordnung und Immunität war weder formell noch, soweit ersichtlich, informell beteiligt.

23 Vgl. Schriftlicher Bericht des Ausschusses für Inneres, Drucksache 3/885, S. 1. 
in den gesamten Materialien unerwähnt bleibt, lässt sich nur spekulieren. Dies könnte an der Zugehörigkeit der beiden Wahlmänner zur KPD liegen; möglicherweise empfand man aber keinen Erläuterungsbedarf, da wegen der zeitlichen Nähe allen Beteiligten die Vorgänge noch bewusst gewesen sein dürften.

\section{Beratung der Gesetzesänderung in der 15. und 16. Wahlperiode}

Angesichts des bei der erstmaligen Anwendung des $\$ 7$ konstatierten Beratungsbedarfs wurden im weiteren Verlauf des Jahres 2004 auf Ausschussebene, das heißt im Wege einer so genannten Selbstbefassung ( $\$ 62$ Abs. 1 Satz 3 GO-BT $)^{24}$, mögliche Präzisierungen des Gesetzes geprüft. In der maßgeblichen Sitzung in Geschäftsordnungsangelegenheiten ${ }^{25} \mathrm{am}$ 2. Dezember 2004 wurde die Beibehaltung eines Immunitätsschutzes für die Mitglieder der Bundesversammlung nicht in Frage gestellt. Insoweit lässt sich anführen, dass auch für das Verfassungsorgan Bundesversammlung die Sicherung der Arbeits- und Funktionsfähigkeit als Schutzzweck und -begründung dienen kann. Ebenso wenig wurden die einfachgesetzliche Ausgestaltung als unzureichend bemängelt und eine Verankerung im Grundgesetz gefordert. Im Vordergrund der Beratungen standen die Dauer, insbesondere der Beginn, der Mitgliedschaft der von den Landesparlamenten Gewählten sowie die Frage, ob der Bundestag zur Entscheidung über Angelegenheiten eines anderen Verfassungsorgans und seiner Mitglieder berufen sein kann und soll ${ }^{26}$.

Die Dauer der Mitgliedschaft der von den Landesparlamenten Gewählten ist eindeutig bestimmbar. Gemäß $\$ 7$ Abs. 4 wird die Mitgliedschaft mit dem Eingang der schriftlichen Annahmeerklärung beim Präsidenten des jeweiligen Landesparlaments erworben. Wird bis zum Ablauf der vom Parlamentspräsidenten gesetzten Frist keine Erklärung abgegeben, gilt die Wahl als zu diesem Zeitpunkt angenommen. Die Mitgliedschaft endet, wie im Hinblick auf die eingeräumten Rechte bereits 1959 in $\$ 9$ Abs. 5 ausdrücklich geregelt, mit der Beendeterklärung der Bundesversammlung durch den Bundestagspräsidenten. Für eine Geltung des Immunitätsschutzes nur während der konkreten Dauer der Bundesversammlung konnte jedenfalls für die seinerzeit geltende Rechtslage die zitierte Begründung im Regierungsentwurf von 1959 sprechen, dass den Mitgliedern der Bundesversammlung „für deren Dauer“ Indemnität, Immunität, Zeugnisverweigerungsrecht und Mandatsschutz gewährt werden sollte. Bei einer derart begrenzten Geltung käme dem Immunitätsschutz, aber auch dem Mandatssschutz und dem Zeugnisverweigerungsrecht praktisch keine Bedeutung zu.

Legt man das Verständnis des Immunitätsschutzes seitens des Bundestages zugrunde (vgl. Nr. 4 der Grundsätze in Immunitätsangelegenheiten - Anlage 6 zur GO-BT), werden

24 Berichterstatter waren die Abgeordneten Erika Simm (SPD), Eckart von Klaeden (CDU/CSU), Volker Beck (Bündnis 90/Die Grünen), Jörg van Essen (FDP).

25 Sitzungen des 1 . Ausschusses finden getrennt nach Geschäftsordnungs- und Immunitätsangelegenheiten statt - ebenso in Wahlprüfungsangelegenheiten bei nur teilweiser Identität der Mitglieder, die gemäß $₫ 3$ Abs. 2 Wahlprüfungsgesetz gewählt und nicht seitens der Fraktionen gemäß $\$ 57$ Abs. 2 GO-BT (nur) benannt werden.

26 Auch im Schrifttum ist die Frage der Zuständigkeit für Immunitätsentscheidungen diskutiert worden, vgl. Christian M. Burkiczak, Die Aufhebung der Immunität von Mitgliedern der Bundesversammlung, in: Verwaltungsblätter für Baden-Württemberg (VBlBW) 2006, S. 90 ff. (mit Nachtrag in: VBlBW 2007, S. 371). 
die Arbeits- und Funktionsfähigkeit der Bundesversammlung sichergestellt. Dies umfasst für das einzelne Mitglied neben der Teilnahme an der Bundesversammlung auch die Vorbereitung und die Anreise. Die Immunität muss daher auch vor Versuchen schützen, die $\mathrm{Zu}-$ sammensetzung der Bundesversammlung zu verändern. So sieht das Bundesverfassungsgericht bei Bundestagsabgeordneten aufgrund des Prinzips der Repräsentation eine Prüfung als erforderlich an, ob Anhaltspunkte vorliegen, dass versucht wird, durch bewusst sachfremde Eingriffe die vom Wähler gewollte Zusammensetzung des Parlaments zu verän$\operatorname{dern}^{27}$.

Auch die anderen entsprechend anwendbaren Rechte sprachen nicht für eine Begrenzung nur auf den Tag der Bundesversammlung. Zwar zielt die Indemnität (Art. 46 Abs. 1 GG) vornehmlich auf die Sitzung selbst ab, indem ein Verfolgungsschutz für Redebeiträge und Abstimmungen statuiert wird. Aber auch insoweit ist eine zeitliche - wie sachlicheAusdehnung gegeben, indem der Schutz entsprechend der vom Bundestag für seinen Bereich vertretenen Auffassung auch vorgelagerte Fraktionssitzungen erfasst. Weiterhin sprechen der Mandatsschutz (Art. 48 Abs. 2 GG, insbesondere Behinderungsverbot) sowie das Zeugnisverweigerungsrecht (Art. 47 GG - Berechtigung zur Zeugnisverweigerung über Personen, die einem Mitglied der Bundesversammlung in dieser Eigenschaft Tatsachen anvertraut haben, oder denen sie in dieser Eigenschaft Tatsachen anvertraut haben, sowie über diese Tatsachen selbst) für einen die gesamte Zeit der Mitgliedschaft erfassenden Geltungszeitraum. Im Ergebnis wurde daher - wie in der Gesetzesbegründung dokumentiert - von einem Immunitätsschutz ab Mitgliedschaftserwerb ausgegangen ${ }^{28}$; eine Klarstellung im Gesetz ist aber nicht für erforderlich gehalten worden.

Zur Frage, welches Organ für Immunitätsangelegenheiten der Mitglieder der Bundesversammlung zuständig sein sollte, kamen vier Lösungsansätze in Betracht: die Bundesversammlung beziehungsweise ein spezielles Gremium, der Bundestagspräsident als Präsident der Bundesversammlung, das jeweilige Landesparlament bezüglich der von diesem Gewählten sowie schließlich der Bundestag.

Für eine Zuständigkeit der Bundesversammlung selbst sprach zunächst der Gesichtspunkt, dass über Angelegenheiten eines Verfassungsorgans nicht von anderer Stelle zu befinden sei. Allerdings tritt die Bundesversammlung erst am Tag der Wahl des Bundespräsidenten zusammen. In der Praxis kämen ihre Entscheidungsmöglichkeiten, da der Immunitätsschutz schon zeitlich früher einsetzt, zu spät; ein Verzicht auf jegliche Immunitätsaufhebung vor diesem Termin würde den Belangen der Strafrechtspflege zuwiderlaufen. Eine Entscheidungsmöglichkeit erst am Wahltag selbst wäre zudem bedeutungslos, da der Schutz bereits am selben Tag wieder entfällt. Sondersitzungen der Bundesversammlung nur zur Entscheidung in Immunitätsangelegenheiten einzuberufen erschien nicht als sachgerechter Ausweg. Ebenso wenig kam ein Unterorgan der Bundesversammlung in Betracht, das auch nicht von der Bundesversammlung selbst rechtzeitig eingesetzt werden könnte. In einer Zuständigkeit des Bundestagspräsidenten wurde ebenfalls keine Abhilfe gesehen. Diesem kommt auch in Immunitätsangelegenheiten der Mitglieder des Bundestages nur eine Notzuständigkeit ( $\$ 107$ GO-BT) vor der Konstituierung des Immunitätsausschusses 
$\mathrm{zu}^{29}$. Dieser ist ansonsten das Organ, das mit den Vorgängen vertraut ist und eine einheitliche Handhabung sicherstellt. Für die Landesparlamente ließ sich geltend machen, dass sie für die jeweiligen Wahlen einschließlich etwaiger Wahlprüfungen zuständig sind und zudem in Immunitätsangelegenheiten ihrer Mitglieder nach Maßgabe der jeweiligen Regelungen befinden. Eine Landtagszuständigkeit hätte jedoch eine Kompetenz über Angelegenheiten eines Bundesorgans begründet, dessen Immunitätsschutz sich nach Bundesrecht bemisst. Zudem wäre nicht auszuschließen gewesen, dass die Entscheidungspraxis angesichts nicht ganz einheitlicher Landesrechte und Verfahrensweisen divergiert und letztlich Ungleichbehandlungen bedingt hätte. Im Ergebnis wurde mangels besserer Alternativen an einer $\mathrm{Zu}$ ständigkeit des Bundestages festgehalten, die auch am ehesten eine Gleichbehandlung der von den Landesparlamenten Gewählten mit den auch der Bundesversammlung angehörenden Bundestagsabgeordneten garantiert. Berücksichtigt wurde außerdem als Parallele die unbestritten angenommene, aber noch nie praktisch gewordene Zuständigkeit des Bundestages für Immunitätsangelegenheiten des Bundespräsidenten gemäß Art. 60 Abs. $4 \mathrm{GG}^{30}$.

Da das sich abzeichnende Beratungsergebnis die von den Landesparlamenten zu wählenden Mitglieder der Bundesversammlung betreffen würde, wurden verabredungsgemäß deren Präsidenten durch Schreiben der Vorsitzenden des 1. Ausschusses, Erika Simm, über den beabsichtigten Regelungsinhalt unterrichtet und eine Stellungnahme ermöglicht. 14 Landesparlamente haben entweder ausdrücklich oder durch Verzicht auf eine Antwort keine Bedenken geltend gemacht. Nur in zwei Antworten wurde gegen eine Zuständigkeitsübertragung an den Bundestag eingewandt, dass grundsätzlich jedes Verfassungsorgan selbst über eigene Angelegenheiten zu entscheiden habe. Da die Bundesversammlung erst am Tage ihres Zusammentritts handlungsfähig sei, wurde vorgeschlagen, entweder eingehende immunitätsrechtliche Aufhebungsersuchen an den Immunitätsausschuss des Bundestages zur Vorbereitung einer von der Bundesversammlung bei Tätigkeitsbeginn zu treffenden Entscheidung weiterzuleiten (so Nordrhein-Westfalen) oder eine Vorentscheidung auf Bundestagsebene zuzulassen, über die die Bundesversammlung aber erneut zu befinden haben sollte (so Bremen). Die Antworten und Stellungnahmen wurden den Berichterstattern zugänglich gemacht; die Änderungsvorschläge sind aber aus praktischen Erwägungen nicht aufgegriffen worden.

Eine abschließende Beratung einer Neuregelung fand wegen des vorzeitigen Endes der 15. Wahlperiode nicht mehr statt. In der 16. Wahlperiode wurden die Beratungen anhand der Unterlagen der 15. Wahlperiode zunächst auf Berichterstatterebene ${ }^{31}$ wieder aufgenommen. In einem entsprechend der Ausschusspraxis vom Vorsitzenden des Ausschusses Thomas Strobl geleiteten Berichterstattergespräch wurde ohne strukturelle Änderung gegenüber dem Beratungsergebnis der 15. Wahlperiode ein Regelungsvorschlag abgestimmt. Dieser fand die einmütige Billigung des Ausschusses in einer Sitzung in Geschäftsordnungsangelegenheiten am 9. Februar 2006. Der auftragsgemäß vom Sekretariat des Ausschusses erstellte und den Obleuten des Ausschusses mit dem Ziel der Einbringung als Fraktionsinitiati-

29 Nach Konstituierung des zuständigen Ausschusses fungiert der Bundestagspräsident laut Bundesverfassungsgericht - etwas überzeichnend - nur als „Poststelle“, BVerfGE 104, S. 310 (S. 324).

30 So zum Beispiel schon Roman Herzog, a.a.O. (Fn. 9); die Bundestagszuständigkeit ist jetzt auch in der Gesetzesbegründung erwähnt - Drucksache 16/3303, S. 3.

31 Abgeordnete Ole Schröder (CDU/CSU), Christine Lambrecht (SPD), Jörg van Essen (FDP), Dagmar Enkelmann (Die Linke), Volker Beck (Bündnis 90/Die Grünen). 
$\mathrm{ve}^{32}$ übermittelte Gesetzentwurf wurde am 8. November 2006 mit geringfügigen redaktionellen Änderungen von den Fraktionen von CDU/CSU, SPD, FDP und Bündnis 90/Die Grünen als Entwurf eines Gesetzes zur Änderung des Gesetzes über die Wahl des Bundespräsidenten durch die Bundesversammlung 33 eingebracht. In der Gesetzesbegründung wurde zusätzlich unterstrichen, dass die Zuständigkeit der Landesparlamente in landesrechtlich begründeten Immunitätsangelegenheiten ihrer in die Bundesversammlung gewählten Mitglieder selbstverständlich unberührt bleibt. Der Gesetzentwurf wurde vom Bundestag in erster Lesung ohne Aussprache an den 1. Ausschuss federführend sowie an den Innen- und den Rechtsausschuss mitberatend überwiesen ${ }^{34}$. Nach einstimmiger Billigung in beiden mitberatenden Ausschüssen votierte der 1. Ausschuss ebenfalls einstimmig auf unveränderte Annahme ${ }^{35}$, die das Plenum in zweiter und dritter Beratung am 10. Mai 2007 - wiederum ohne Debatte - einstimmig beschloss ${ }^{36}$.

\section{Schließung einer Gesetzeslücke und Klarstellung für die Zukunft}

Abgesehen von der Klärung der Zuständigkeitsfrage schließt die Änderung des Gesetzes eine Lücke, die sich beim ersten Anwendungsfall gezeigt und seinerzeit zur bereits erwähnten Auflage veranlasst hatte. Indem $\$ 7$ Abs. 1 Satz 2 2. Halbsatz ausdrücklich anordnet, dass die vom Bundestag oder seinem zuständigen Ausschuss erlassenen Regelungen in Immunitätsangelegenheiten entsprechend gelten, sind neben $\$ 107$ GO-BT insbesondere die generelle Genehmigung zur Aufnahme von Ermittlungen sowie die Immunitätsgrundsätze des Ausschusses für Wahlprüfung, Immunität und Geschäftsordnung (vgl. Anlage 6 zur GO-BT) anzuwenden. So sind beim Präsidenten des Bundestages eingehende Schreiben einer Staatsanwaltschaft unmittelbar an den zuständigen Immunitätsausschuss weiterzuleiten ( $\$ 107$ Abs. 1 GO-BT). Aufgrund des Beschlusses des Bundestages betreffend die Aufhebung der Immunität seiner Mitglieder setzt die Aufnahme von Ermittlungen wegen Straftaten mit Ausnahme politischer Beleidigungen künftig keine Einzelfallgenehmigung durch den Bundestag voraus. Es reicht eine Mitteilung an den Bundestagspräsidenten, die intern an den Immunitätsausschuss weitergeleitet wird. Die Ermittlungen dürfen grundsätzlich 48 Stunden nach Eingang des Antrags aufgenommen werden. Einer Plenarentscheidung bedarf es erst, wenn Anklage erhoben, ein Strafbefehl erlassen oder eine Durchsuchung beim Beschuldigten erfolgen soll. Insbesondere für Strafbefehle aufgrund von Verkehrsdelikten kommt bei Einstimmigkeit im Immunitätsausschuss eine so genannte Vorentscheidung des Ausschusses in Betracht, die als Entscheidung des Bundestages gilt, sofern ihr nicht binnen sieben Tage nach Verteilung widersprochen wird (vgl. Nummern 3 Abs. 1 und 7 des oben genannten Beschlusses) ${ }^{37}$.

32 Eine Nutzung des ausdrücklichen Rechts des 1. Ausschusses, zu Fragen aus seinem Geschäftsbereich dem Bundestag Empfehlungen vorzulegen ( $\$ 128$ GO-BT), ist hier nicht erwogen worden, da die angestrebte Regelung eines Gesetzes bedurfte.

33 Drucksache 16/3303.

34 Stenografisches Protokoll der 70. Sitzung vom 3. November 2006, S. 6911 D.

35 Drucksache 16/5096.

36 Stenografisches Protokoll der 97. Sitzung, S. 9852 C.

37 In der Praxis hat es in diesem 1951 geschaffenen und später auch auf andere Konstellationen ausgedehnten Verfahrensweg noch nie einen Widerspruch gegeben. 
Als vom Bundestag erlassene und den Immunitätsschutz konkretisierende Regelungen sind des Weiteren Bestimmungen zur Rechtshilfe in Strafsachen anzusehen. So finden gemäß $\$ 77$ Abs. 2 des Gesetzes über die Internationale Rechtshilfe in Strafsachen ${ }^{38}$ auf bei deutschen Stellen eingehende ausländische Rechtshilfeersuchen die Vorschriften zur Immunität, zur Indemnität und die Genehmigungsvorbehalte für Durchsuchungen und Beschlagnahmen in den Räumen eines Parlaments Anwendung, welche für deutsche Straf- und Bußgeldverfahren gelten ${ }^{39}$.

Schließlich stellt die Begründung klar, dass die Zugehörigkeit der Bundestagsabgeordneten zur Bundesversammlung keine immunitätsrechtliche Doppelbehandlung zur Folge haben soll. Ihr Status in der Bundesversammlung unterscheidet sich zwar nicht von demjenigen der von den Landesparlamenten Gewählten. Es reichen aber jeweils immunitätsrechtliche Schritte mit Bezug auf die Mitgliedschaft im Bundestag aus. Die Staatsanwaltschaften haben also nicht jeweils getrennt nach Zugehörigkeit zum Bundestag und zur Bundesversammlung über die beabsichtigte Einleitung von Ermittlungen zu berichten oder Aufhebungsanträge zu stellen. Andernfalls würde sich die nur schwer zu beantwortende Frage stellen, ab wann die Mitgliedschaft der Bundestagsabgeordneten in der Bundesversammlung beginnt ${ }^{40}$.

38 Zunächst eingefügt im Rahmen der Ausschussberatungen zum Entwurf eines Europäischen Haftbefehlsgesetzes (vgl. Drucksache 15/2677, S. 4 und 6). Nach Nichtigerklärung des Gesetzes vom 21. Juli 2004 (BGBl. I, S. 1748) durch Urteil des Bundesverfassungsgerichts vom 18. Juli 2005, Az.: 2 BvR 2236/04 (BVerfGE 113, S. 273 ff.), enthält das nachfolgende Gesetz vom 20. Juli 2006 (BGBl. I, S. 1721) eine textgleiche Regelung (vgl. auch Drucksache 16/1024 unter Bezugnahme auf Begründungen zum Entwurf des Vorläufergesetzes bezüglich unverändert übernommener Regelungen).

39 Bei Ersuchen des Internationalen Strafgerichtshofes um Überstellung oder sonstige Rechtshilfe gegen einen Bundestags- oder Landtagsabgeordneten ist der jeweilige Parlamentspräsident zu unterrichten, vgl. $\$ 70$ des Gesetzes zur Ausführung des Römischen Statuts des Internationalen Strafgerichtshofes vom 17. Juli 1998 vom 21. Juni 2002 (BGBl. I, S. 2144).

40 Christian M. Burkiczak, a.a.O. (Fn. 26), S. 92, tendiert in dieser Frage zum Zeitpunkt des Zusammentritts.

\section{Wie wichtig ist die Person? Zur Bedeutung von Persönlichkeitsfaktoren von Wahlkreisbewerbern bei Bundestagswahlen ${ }^{*}$}

\section{Christian Mackenrodt}

Er müht sich redlich: Er ist mit seinem Stand überall im Wahlkreis präsent. Er trotzt Wind, Wetter und der Hast der Passanten, die sich mehr für seine Kugelschreiber als für seine Person interessieren. Er verschuldet sich für den Wahlkampf und opfert der Politik die Freizeit mit seiner schwangeren Freundin. Am Ende verliert er gegen den übermächtigen SPD-

\footnotetext{
Ich danke Joachim Behnke, Thorsten Faas und Rüdiger Schmitt-Beck für wertvolle Anregungen zu diesem Artikel.
} 